\title{
Seeing faces in the noise: Stochastic activity in perceptual regions of the brain may influence the perception of ambiguous stimuli
}

\author{
HEATHER A. WILD and THOMAS A. BUSEY \\ Indiana University, Bloomington, Indiana
}

\begin{abstract}
Research on binocular rivalry and motion direction discrimination suggests that stochastic activity early in visual processing influences the perception of ambiguous stimuli. Here, we extend this to higher level tasks of word and face processing. In Experiment 1, we used blocked gender and word discrimination tasks, and in Experiment 2, we used a face versus word discrimination task. Stimuli were embedded in noise, and some trials contained only noise. In Experiment 1, we found a larger response in the N170, an ERP component associated with faces, to the noise-alone stimulus when observers were performing the gender discrimination task. The noise-alone trials in Experiment 2 were binned according to the observer's behavioral response, and there was a greater response in the N170 when they reported seeing a face. After considering various top-down and priming-related explanations, we raise the possibility that seeing a face in noise may result from greater stochastic activity in neural faceprocessing regions.
\end{abstract}

A basic goal of cognitive neuroscience is to link behavior with neural mechanisms. Two notable successes have come from research on binocular rivalry and motion direction discrimination, in which an ambiguous stimulus is presented and physiological correlates are found between the reported percept and ongoing activity in visual areas such as V1 and MT (Britten, Newsome, Shadlen, Celebrini, \& Movshon, 1996; Britten, Shadlen, Newsome, \& Movshon, 1993), as well as in extrastriate areas (Tong, Nakayama, Vaughan, \& Kanwisher, 1998). In the present work, we seek to generalize this principle to the domains of word and face processing, which also may involve specialized neural areas in the inferotemporal cortex (Kanwisher, Stanley, \& Harris, 1999; but see also Gauthier, Tarr, Anderson, Skudlarski, \& Gore, 1999, for evidence of expertise effects in the same area). Whereas prior work has been done with single-cell and fMRI recording, here we rely on known response properties of electrophysiological (EEG) measures.

In the present study, we address the relation between face-related brain activity and the reported percept by embedding faces and words in noise and using noisealone displays to create an ambiguous stimulus. We will rely on the N170 component of the event-related potential (ERP) $)^{1}$, which has been linked to activity in face-

This research was supported by an NIH grant to T.A.B. The authors thank Shlomo Bentin and two anonymous reviewers for their helpful comments during the review process and Jason Gold for helpful discussions about added-noise procedures. More information is available at www.indiana.edu/ busey/. Correspondence concerning this article should be addressed to H. A. Wild or T. A. Busey, Department of Psychology, Indiana University, Bloomington, IN 47405 (e-mail: hwild@indiana.edu or busey@indiana.edu). related regions of the human. Jeffreys (1989) has shown that Mooney faces elicit a negative-going component that occurs $170 \mathrm{msec}$ after stimulus onset. However, when these faces are inverted, they are difficult to interpret as a face, and the N170 is likewise attenuated. Numerous subsequent studies have also shown that faces elicit a strong N170 component (Bentin, 1997; Bentin, Allison, Puce, Perez, \& McCarthy, 1996; Olivares \& Iglesias, 2000). This downward deflection is largest over the temporal lobes (Bentin et al., 1996). Although there is some disagreement as to the precise neural locus of the N170, its latency and spatial location suggest that it represents activity in regions typically associated with early perceptual processing of faces and other complex visual stimuli.

In addition to these bottom-up factors, perceptual expertise and context modulate the N170. Tanaka and Curran (2001) found a larger N170 component in bird and dog experts for faces of animals on which an individual was an expert. Rossion, Gauthier, Goffaux, Tarr, and Crommelinck (2002) trained observers to individuate novel objects called greebles, and found expertise effects in the N170. To demonstrate contextual effects, Bentin and colleagues (Bentin \& Golland, 2002; Bentin, Sagiv, Mecklinger, Frederici, \& von Cramon, 2002) presented pairs of dots that evoked only a weak N170 response to observers. The dots were subsequently shown surrounded by face features that made them interpretable as eyes. After priming with a face context, the $\mathrm{N} 170$ response to the dots alone increased. These studies demonstrate that context interacts with perceptual information to modulate the N170.

In the present study, we examine the link between the activity reflected by the $\mathrm{N} 170$ and the behavioral response in a task that has been made ambiguous with respect to faces and words. We recorded EEGs from observers 
while they viewed faces and words embedded in random pixel noise, and some trials contained only the noise as an ambiguous stimulus. In the first experiment, observers completed a word discrimination task (honesty vs. trust) and a gender categorization task. This extended prior work on contextual influences on the N170 by examining the effect of observer expectations on the EEG response to a stimulus without readily interpretable facelike features (i.e., the noise-alone display). In Experiment 2, we presented the same stimuli to observers but intermixed the trials so that the observers made a face versus word judgment on each trial. Thus, the observers had no reason to expect a face versus a word on any particular trial. The question was whether we would see modulation of the N170 without the influence of expectations and bottom-up perceptual information.

\section{EXPERIMENT 1}

\section{Method}

Participants. Nine right-handed observers participated in the experiment. These observers were students at Indiana University, whose participation constituted part of their labwork or coursework. All the observers were naive as to the purpose of the experiment.

Apparatus. The EEG was sampled at $1000 \mathrm{~Hz}$ and was amplified by a factor of 20,000 (Grass amps model P511K) and bandpass filtered at $0.1-100 \mathrm{~Hz}$ (notch at $60 \mathrm{~Hz}$ ). Signals were recorded from sites $\mathrm{F} 3, \mathrm{~F} 4, \mathrm{Cz}, \mathrm{T}$, and $\mathrm{T} 6$, with a nose reference and forehead ground; all channels had below 5-kOhm impedance. Recording was done inside a Faraday cage. Eyeblink trials were identified from a characteristic signal in channels F3 and F4 and were removed from the analysis with the help of blink calibration trials. Images were shown on a 21-in. (53.34-cm) Macintosh color monitor approximately $44 \mathrm{in} .(112 \mathrm{~cm})$ from the participants.

Stimuli. The entire stimulus set appears in Figure 1. Face stimuli consisted of grayscale frontal views of one male and one female face with neutral expressions, generated using Poser (Metacreations). The faces subtended a visual angle of $2.1^{\circ} \times 2.8^{\circ}$. Two lowimagery words were chosen for the second task (honesty and trust). The words subtended a visual angle of $1.1^{\circ} \times .37^{\circ}$. All the stimuli were embedded in white noise $\left(4.33^{\circ} \times 4.33^{\circ}\right.$ of visual angle) that was identical (i.e., not resampled) on all the trials. This single noise field was uniform white noise with a mean luminance of $30 \mathrm{~cd} / \mathrm{m}^{2}$ and a standard deviation of $14.5 \mathrm{~cd} / \mathrm{m}^{2}$. This noise was added to the faces and words on a pixel-by-pixel basis. The faces and words had standard deviations of 7.0 and $1.7 \mathrm{~cd} / \mathrm{m}^{2}$ at full contrast prior to the addition of the noise. To create the low-contrast versions, the contrast of the faces and words was adjusted until independent raters judged that they were near threshold and approximately equally detectable. The standard deviations of the low-contrast faces and words were 1.27 and $0.52 \mathrm{~cd} / \mathrm{m}^{2}$, respectively.

Procedure. The observers completed blocks of trials for face discrimination and word discrimination tasks. The observers freely viewed the stimulus, and although no fixation point was used, the stimulus appeared in the same location on each trial and was framed by the edge of the monitor. For the gender discrimination task, the observers had to indicate whether the face was male or female, and for the word discrimination task, the observers had to indicate whether the word was honesty or trust. They were told that there was a stimulus present on every trial, despite the fact that one third of the trials were noise alone. The observers were also told that faces and words would appear equally often.

Stimuli were presented for $1,000 \mathrm{msec}$. EEG was recorded from $100 \mathrm{msec}$ prior to stimulus onset to $1,100 \mathrm{msec}$ poststimulus onset. There were 100 trials with a word or a face at each contrast level and 200 noise-alone trials, for a total of 600 trials. The observers responded after each trial via a numeric keypad.

\section{Results and Discussion}

The data from Experiment 1 are shown in Figure 2. Consider first the thin lines, which are the responses to the high-contrast faces and words. The amplitude of the N170 is greater for high-contrast faces than for highcontrast words for both electrode sites. The thick light-
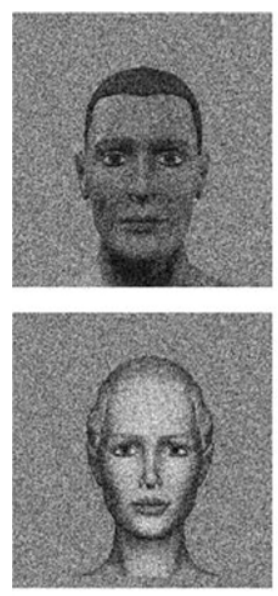

High-Contrast Faces
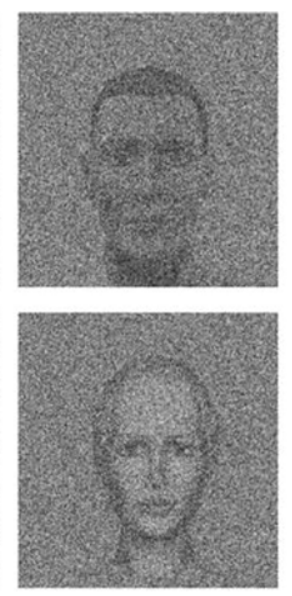

Low-Contrast Faces
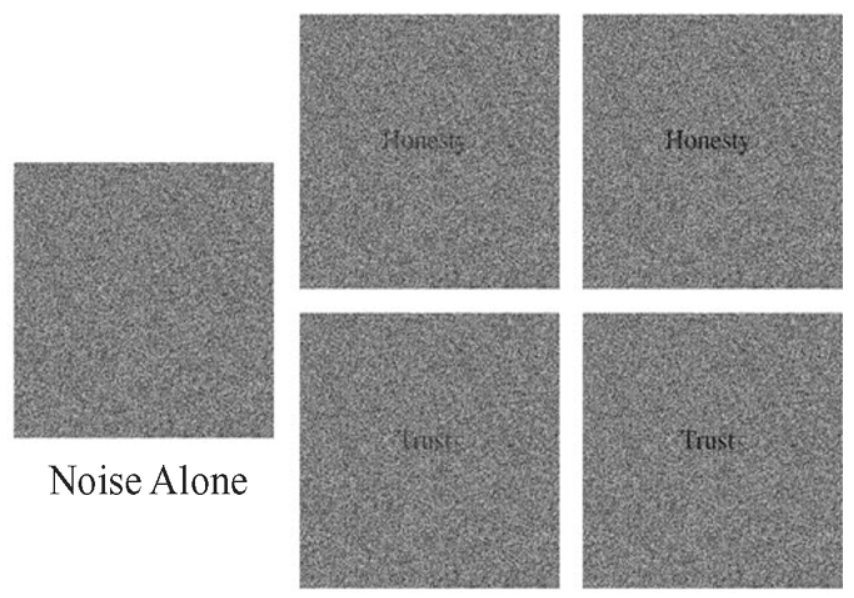

Low-Contrast Words

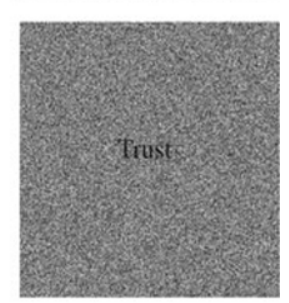

High-Contrast Words

Figure 1. Stimuli used in Experiments 1 and 2. For Experiment 1, high- and low-contrast faces were intermixed with noise-alone trials in a gender identification task, and high- and low-contrast words were intermixed with noise-alone trials in a word identification task. In Experiment 2, both faces and words were intermixed with noise-alone trials in a face/word categorization task. The low-contrast versions have been enhanced for publication, and the original stimuli can be seen at http://cognitrn.psych.indiana.edu/busey/FacesinNoise/. 

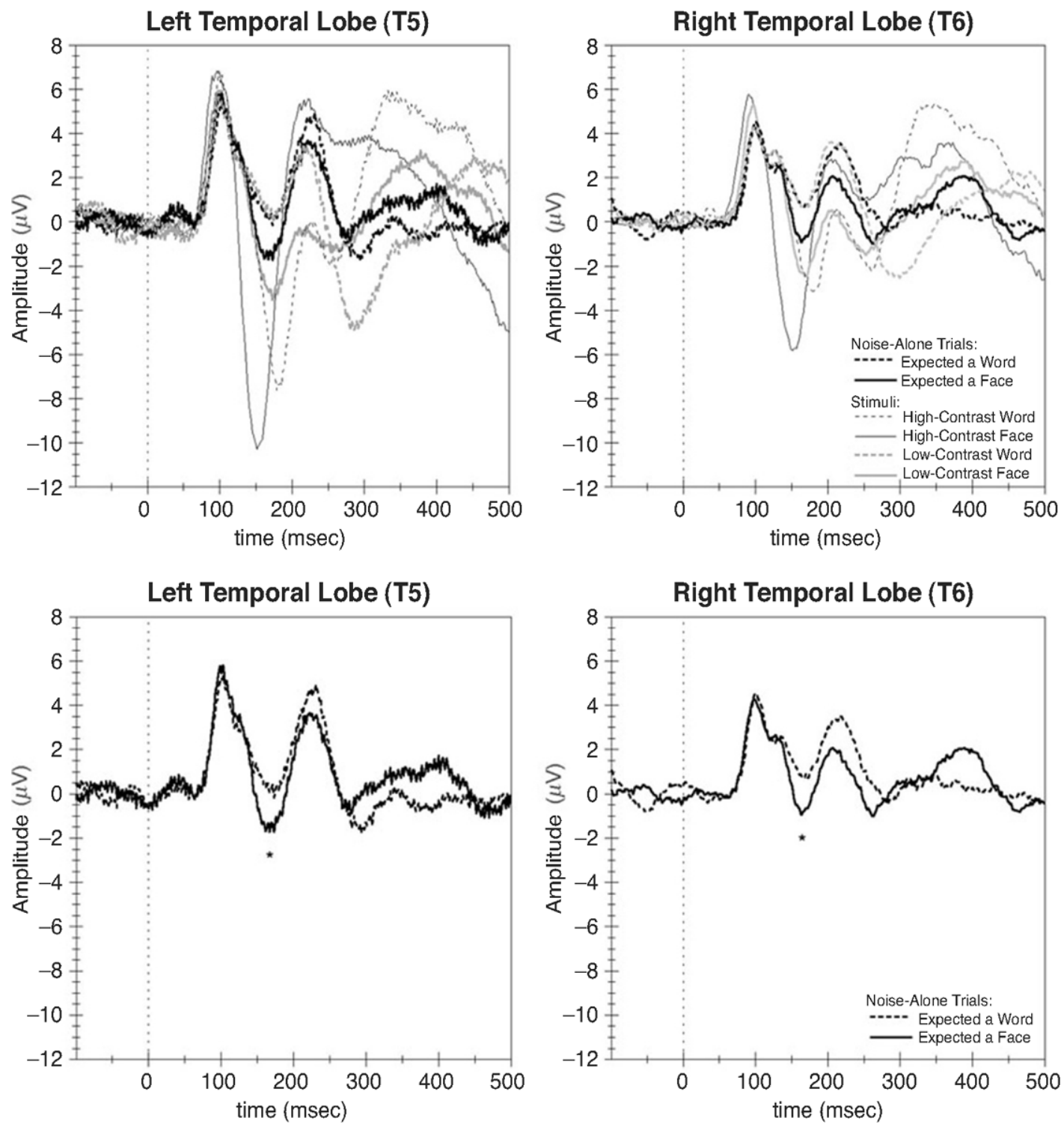

Figure 2. Data from Experiment 1: event-related potentials elicited at temporal lobe sites T5 (left panel, left hemisphere) and T6 (right panel, right hemisphere). All data are shown in the top panel; darker lines indicate noise-alone trials. These data are shown alone in the bottom panels. The asterisks in the left and right lower panels indicate significant differences between the two responses at the N170 component in both channels. The N170 component is the large dip that occurs between 100 and 200 msec.

gray lines, corresponding to the low-contrast condition, show this same pattern. These data show that N170 differentiates between faces and words. The dark curves, highlighted in the lower panels of Figure 2, come from the noise-alone trials. The solid and dashed thick curves come from blocks in which the observer expected a face or a word, respectively. In both channels, the amplitude ${ }^{2}$ of the N170 is significantly greater for noise-alone trials when the observers are expecting a face rather than a word for electrode sites T5 [paired two-tailed $t(8)=$ $2.62, p<.05]$ and T6 $[t(8)=2.35, p<.05]$.

These results extend the findings of Bentin's studies (Bentin \& Golland, 2002; Bentin et al., 2002) to stimuli that contain no face-like features. Most important, the results of Experiment 1 show that the N170 can be modulated by the task of looking for a face, and not just by the physical presence of a face or face-like features. The next step was to use the N170 to address how activity might be related to a behavioral response when we removed contextual information as well. This was the aim of Experiment 2, which was identical to Experiment 1, except that we used a mixed design and had the observers complete a face versus word discrimination task. The central question of Experiment 2 was whether, on noise-alone trials, the observers would produce a larger N170 response when they reported seeing a face. 


\section{EXPERIMENT 2}

\section{Method}

Participants. Ten right-handed observers participated in the experiment.

Procedure. All the stimuli in Figure 1 were presented in random order, and the observers had to indicate whether a face or a word was embedded in the noise. They were told that there was a stimulus present on every trial, despite the fact that one third of the trials were noise alone. The observers were also told that faces and words would appear equally often. The observers responded via a joystick, using a single finger, and were asked to make speeded responses, which was intended to eliminate additional guessing strategies not tied to the initial perceptual processing of the stimulus. There were 120 trials with a word or a face at each contrast level and 240 noisealone trials, for a total of 720 trials.
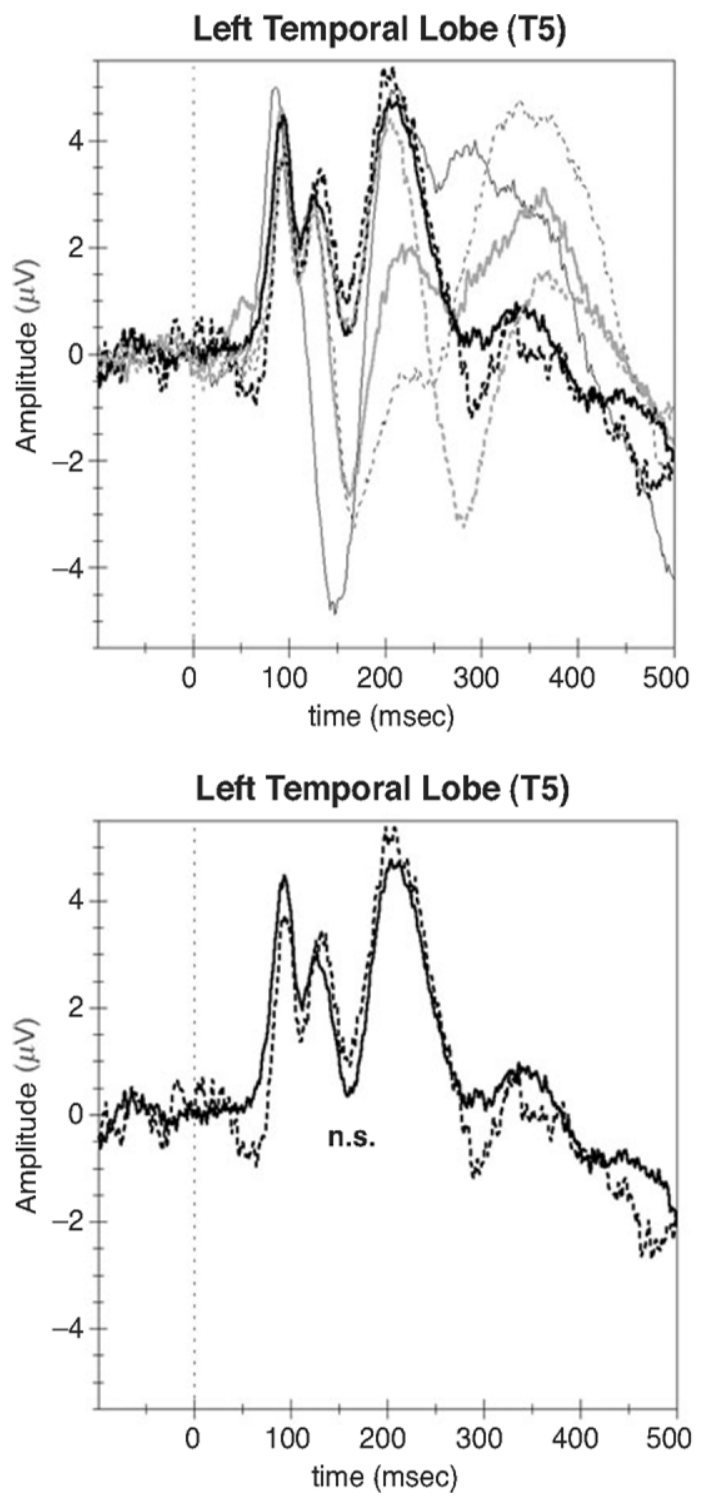

\section{Results and Discussion}

EEG signals were averaged across trials for each participant on the basis of the stimulus, and the noise-alone trials were binned according to the participant's response (either face or word). Figure 3 shows the data for Experiment 2 . The thin curves plot the data for the high-contrast faces and words. As in Experiment 1, we found a larger N170 response for the high-contrast face than for the high-contrast word.

The data that bear on the central question of the experiment came from the trials in which only noise was presented, because on these trials the physical stimulus was held constant and only the response of the observer changed. These data are plotted as thick lines in the lower
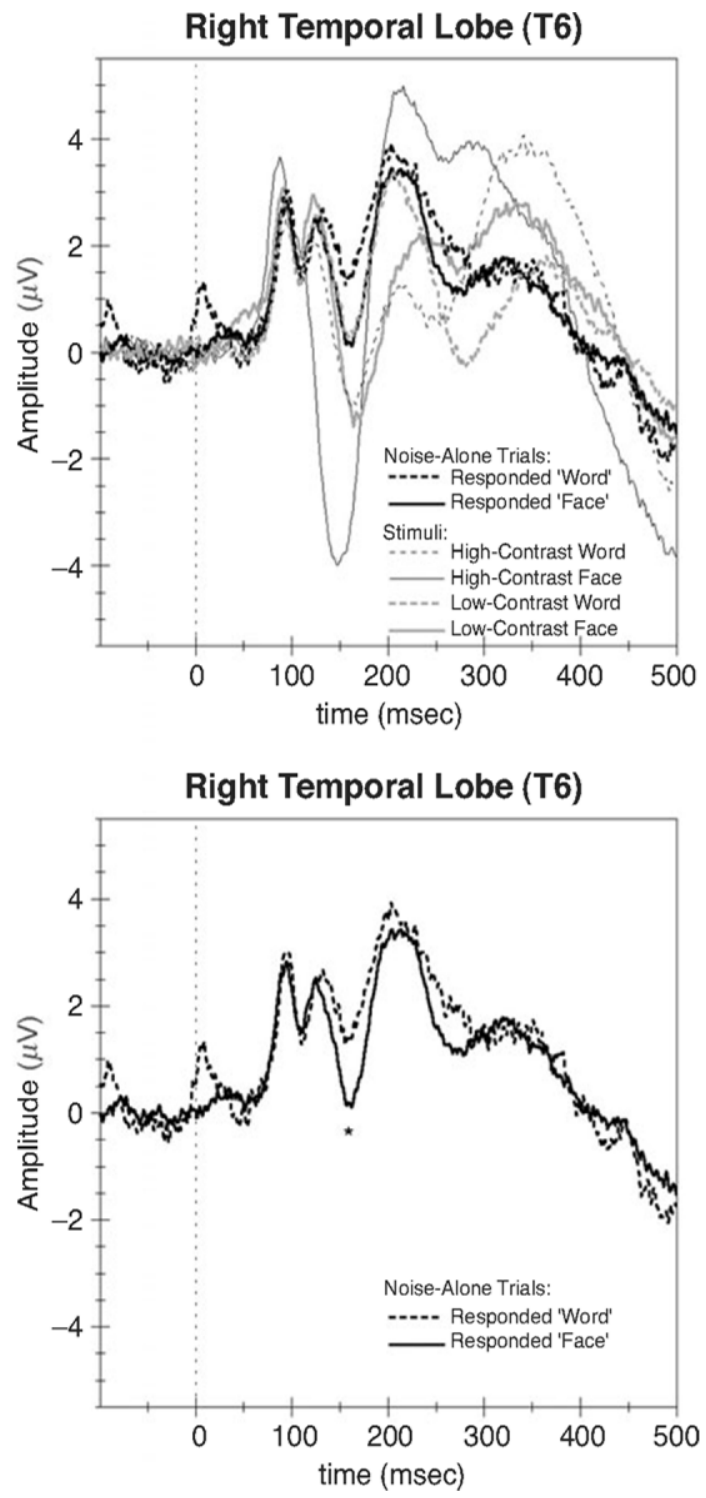

Figure 3. Data from Experiment 2: event-related potentials elicited at temporal lobe sites T5 (left panel, left hemisphere) and T6 (right panel, right hemisphere). All data are shown in the top panel; darker lines indicate noise-alone trials. These data are shown in the bottom panels. The N170 component is the large dip that occurs between 100 and $200 \mathrm{msec}$. The asterisk in the lower right panel indicates significant differences between the two responses at the N170 component. The abbreviation n.s. indicates no significant difference. 
panels of Figure 3. As is shown in the lower right panel of Figure 3, the N170 at the right temporal channel (T6) associated with a face response to the noise-alone stimulus is significantly larger than the N170 associated with a word response [two-tailed, $t(9)=2.74, p<.05$ ].

For the left temporal channel (T5), shown in the lower left panel of Figure 3, this difference is present but not significant $[t(9)=1.54$, n.s.]. Note that in T5, the difference between the N170 amplitudes for the high-contrast words and faces is much smaller than that in channel T6. This is consistent with other right-hemisphere laterality effects involving faces (Farah, 1990).

We also analyzed the P100 and P300 components by averaging the amplitudes in the 80 - to 130 -msec and 260 to $340-\mathrm{msec}$ windows and found no significant differences for either channel, nor in the other three channels. Thus the differences in the ERPs between face and word responses to the noise are confined to the right temporal lobe at about $170 \mathrm{msec}$ after stimulus onset.

The observers were extremely accurate at discriminating high- $(M=99 \%)$ and low- $(M=97 \%)$ contrast faces and words. As can be seen in Table 1, there was a modest bias to respond face to the noise-alone trials, so that the observers made this response $62 \%$ of the time. Since there was a wide range in bias among the observers (11\%-97\%), we examined whether the effect seen in the N170 was related to this bias. Effect size was defined as the difference of the average amplitude in the 150- to 200-msec window for word versus face responses. These data appear in Table 1; note that the effect is present for 9 out of $10 \mathrm{ob-}$ servers. Bias was modestly correlated with effect size $\left(r^{2}=.40, p<.05\right)$. However, further analyses show that this correlation is driven by 3 observers with strong biases. When we remove these observers, the correlation is no longer significant $\left(r^{2}=.10\right)$, but the difference in the N170 for word versus face response trials is still significant $[t(6)=2.69, p<.05]$. Thus, although there appear to be some individual differences in the response properties of the perceptual regions as indexed by the N170, the main results cannot be completely attributed to observer bias to respond face.

Table 1

Probability of Responding Face to a Noise-Alone Stimulus and Effect Size Data for All Observers

\begin{tabular}{cc}
\hline$P($ "face" $)$ & Effect Size $(\mu \mathrm{V})$ \\
\hline .11 & 2.78 \\
.29 & 7.60 \\
.43 & 6.21 \\
.47 & 2.71 \\
.60 & -2.06 \\
.67 & 6.20 \\
.72 & 0.90 \\
.93 & 17.83 \\
.96 & 33.12 \\
.97 & 15.38 \\
\hline
\end{tabular}

Note-Effect size is computed by subtracting the N170 amplitude to face responses from the N170 amplitude to word responses. Positive numbers imply a greater N170 when observers reported seeing a face.
Table 2

Median Reaction Times (in Milliseconds) Averaged Across Participants for Experiment 2

\begin{tabular}{lccccc}
\hline & \multicolumn{4}{c}{ Response } \\
\cline { 2 - 3 } \cline { 5 - 6 } \multicolumn{1}{c}{ Stimulus } & Average & $S E$ & & Average & $S E$ \\
\cline { 2 - 3 } \cline { 5 - 6 } & 615 & 20 & & 615 & 23 \\
Noise Alone & & & & \\
Face Presented & 508 & 15 & & \\
$\quad$ Low Contrast & 488 & 14 & & \\
$\quad$ High Contrast & & & & 569 & 17 \\
Word Presented & & & & 506 & 13 \\
$\quad$ Low Contrast & & & & \\
$\quad$ High Contrast & & & & & \\
\hline
\end{tabular}

Note-The averages and standard errors are shown for noise-alone stimuli binned by response and for correct responses to high- and lowcontrast faces and words.

The averages of the observers' median reaction times (RTs) for the different conditions appear in Table 2. Faces and words differ on many dimensions, and this may have contributed to the RT differences at high and low contrast. However, the noise-alone trials contained the same stimulus, which makes the comparison between the word response and the face response trials reasonable. For these trials, there was no difference in RTs.

The N170 occurred too early to simply be a signature of the observer's response after it had been executed; thus, although it is possible that the N170 neurons influenced the decision, it is unlikely that the subject's decision influenced the electrophysiological response at $170 \mathrm{msec}$. However, possible pretrial influences also existed, such as priming from the previous trial or, perhaps, expectations that a particular stimulus was going to appear (e.g., the gambler's fallacy). We explored this possibility by examining whether the presentation of a face on the previous trial resulted in a larger N170 on current noise-alone trials. Figure 4 shows the ERPs binned according to each possible response to noise-alone trials (i.e., face or word). These are also conditioned on whether the stimulus presented on the previous trial was a face or a word, so that there are four ERP traces shown. Consider first the two thick curves, which represent trials in which the observer responded face. There is clearly no effect of the prior trial stimulus, since the two curves are almost identical throughout the time period of interest (140-200 msec).

The thin curves in Figure 4 correspond to trials in which the observer responded word. The difference in the amplitude between 140 and $200 \mathrm{msec}$ is significant $[t(9)=$ $3.24, p=.01]$. However, the differences occur late in the window and are small compared with the overall main result, which can be recovered by comparing the average of the thin lines with the average of the thick lines. Furthermore, research shows that there appears to be little effect of prior exposure of the face on the N170 response (Cauquil, Edmonds, \& Taylor, 2000). Given that the effects of the prior trial stimulus are small relative to the overall effects and are limited to trials in which the observer responds word, we rule out the prior trial priming hypothesis as a major explanation of the results. 


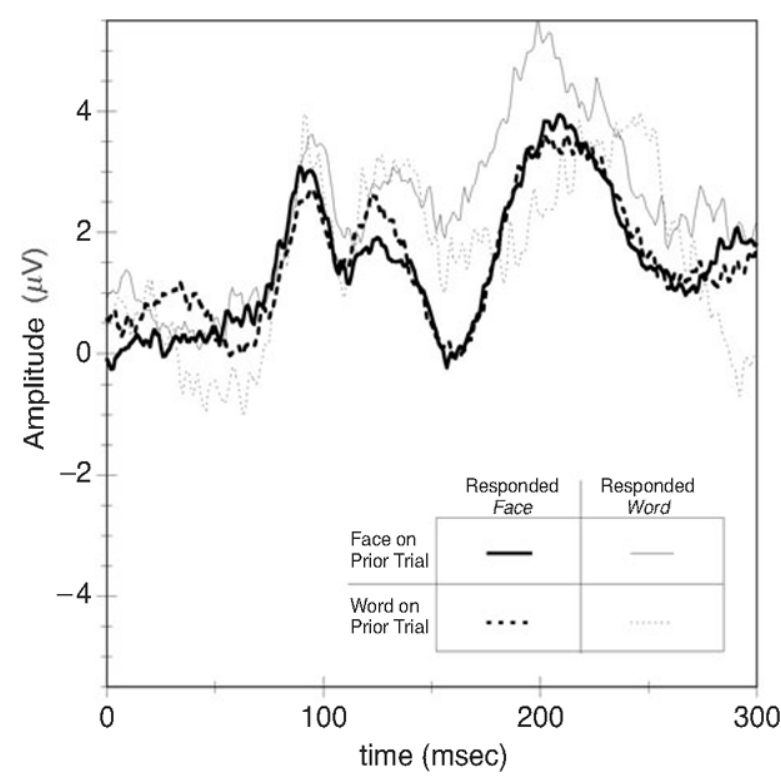

Figure 4. Data from Experiment 2, channel T6 (right hemisphere: event-related potential traces from noise-alone trials conditioned on prior trial and response. Note that the time scale is different from that in the prior figures to emphasize effects at the N170.

\section{GENERAL DISCUSSION}

In the present experiments, we established a relationship between face-related activity, as indexed by the N170, and the eventual behavioral response. Experiment 1 allowed us to extend the work of Bentin and colleagues by eliminating structured face-like features from the stimulus and examining the influence of observer expectations on the N170. In Experiment 2, we found that observers showed a greater N170 when they thought that they saw a face in the noise rather than a word, an effect that was localized to the right temporal lobe around $170 \mathrm{msec}$ post stimulus onset. This result cannot be linked to observer expectations, as in Experiment 1, because we intermixed face and word trials and eliminated any possible stimulusdriven influences, since the conditions we were comparing used trials that contained identical noise. What might produce this result in the N170? Having ruled out explanations based on prior trial priming and response bias, we consider other possible top-down influences, such as fluctuations in attention to different types of information in the image, as well as mechanisms not related to topdown or bottom-up processes, such as stochastic fluctuations in neural activity in the perceptual regions. Both interpretations are interesting, and below we will evaluate the evidence for each possibility.

The observer's decision may be influenced by the nature of the information that is acquired, perhaps through tuning of spatial frequency channels or attention to different face-like features in the noise. Faces tend to have lower spatial frequencies than do words, and observers may tune their spatial frequency filters to one range or another on a given trial. Neurons that respond to faces may receive input from more cells that are tuned to lower spatial frequencies and have a larger receptive field, and this may provide a stronger response at the N170 if observers attend to lower spatial frequencies on noise-alone trials. Evidence that the N170 is sensitive to spatial frequency information comes from Goffaux, Gauthier, and Rossion (2003), who recorded EEG to low- and high-pass-filtered faces and cars. They found that the stronger N170 response for faces is specific to low-pass-filtered stimuli.

There are several pieces of evidence that argue against an explanation of our effects based on dynamic tuning of spatial frequency filters. First, there is no evidence of a preferential response to low-pass-filtered faces during intracranial recording (McCarthy, Puce, Belger, \& Allison, 1999), and numerous studies have shown that line drawings of faces elicit an N170 just as strongly as a real face (e.g., Bentin et al., 2002). Second, studies have shown that attention to specific spatial frequency gratings causes increased positivity in the ERP in the 100- to 200 -msec range (i.e., around the N170) and that this effect is the same regardless of the spatial frequency of the attended stimulus (Bass, Kenemans, \& Mangun, 2002; Martinez, Di Russo, Anllo-Vento, \& Hillyard, 2001). Thus, attending to low spatial frequency bands does not automatically produce a larger N170. Third, the observers in the experiment had no motivation to alter their spatial frequency tuning on a trialby-trial basis. Thus, although we cannot rule out all possible strategies that observers may employ, we have controlled for as many as we can by using a mixed design and have eliminated others by looking at the prior trial priming hypothesis.

Having ruled out as many top-down and prior-trial hypotheses as possible, we would like to raise the intriguing possibility that the behavioral response is directly related to the activity in the N170 neurons. The timing and the spatial location of the N170, as well as the tasks that modulate it, all suggest that the $\mathrm{N} 170$ reflects the neural processing of complex perceptual information. On the basis of this, one possible interpretation of the present data is that the N170-generating neurons influence the response, perhaps by increasing the evidence in favor of a face on the current trial. The idea that activity in a perceptual region can influence the response to an ambiguous stimulus is consistent with the mechanisms involved in binocular rivalry and motion detection. For example, Blake and Logothetis (2002) have suggested that periods of left- and right-eye dominance are governed by a stochastic process with an unstable time constant. A similar principle may be at work here, so that internal stochastic activity combines with neural activity generated by the stimulus to yield the percept. Although this stochastic activity may be quite weak, it may be sufficient to bias the response in favor of one alternative or the other in the absence of bottom-up or top-down evidence.

It should be noted that studies relating the perception of motion in an ambiguous stimulus to biases in MT have shown that the biases appear around 50-100 msec (Brit- 
ten et al., 1993), so although our effects occurred early in processing, the differences at $170 \mathrm{msec}$ may have resulted from feedback from areas involved in higher level tasks. Such top-down effects have been noted with imagery and voluntary attention (O'Craven \& Kanwisher, 2000; Wojciulik, Kanwisher, \& Driver, 1998). However, the mixed design of Experiment 2 provided no incentive for feedback that might have begun prior to the trial. In addition, we found no differences in the frontal electrodes or at earlier time intervals prior to the N170, and so this explanation seems unlikely. It is possible that recurrent feedback from higher cortical areas might occur within a single trial (Hochstein \& Ahissar, 2002), in which these later areas categorize the stimulus and then retune the face-processing areas. This account is not inconsistent with the stochastic activity account but places the locus of the stochastic activity in higher cortical areas. If this view is accurate, it would require a reinterpretation of the N170 as reflecting not only perceptual information, but also feedback from other cortical areas that enable rapid retuning of the response properties of the N170 neurons within a trial. Although we currently have no evidence in favor of or against this explanation, it remains an interesting interpretation.

The use of an ambiguous noise field allows us to establish a link between activity in perceptual regions and the reported percept in the absence of physical differences in the stimuli. By controlling the physical stimulus as much as possible, this technique avoids many of the stimulus differences present in experiments that initially described the response properties of the N170. This methodology can be generalized to other comparisons to reveal how ongoing neural activity affects perception and to delineate how components of the ERP reflect aspects of processing on the pathway from sensation to response.

\section{REFERENCES}

Bass, J. M. P., Kenemans, J. L., \& Mangun, G. R. (2002). Selective attention to spatial frequency: An ERP and source localization analysis. Clinical Neurophysiology, 113, 1840-1854.

Bentin, S. (1997). Separate modules for face perception and face recognition: Electrophysiological evidence [Abstract]. Journal of Psychophysiology, $\mathbf{8 1}$.

Bentin, S., Allison, T., Puce, A., Perez, E., \& McCarthy, G. (1996). Electrophysiological studies of face perception in humans. Journal of Cognitive Neuroscience, 8, 551-565.

Bentin, S., \& Golland, Y. (2002). Meaningful processing of meaningless stimuli: The influence of perceptual experience on early visual processing of faces. Cognition, 86, B1-B14.

Bentin, S., Sagiv, N., Mecklinger, A., Frederici, A., \& von CraMON, Y. D. (2002). Priming visual face-processing mechanisms: Electrophysiological evidence. Psychological Science, 13, 190-193.
Blake, R., \& Logothetis, N. (2002). Visual competition. Nature Reviews Neuroscience, 3, 1-11.

Britten, K. H., Newsome, W. T., Shadlen, M. N., Celebrini, S., \& Movshon, J. A. (1996). A relationship between behavioral choice and the visual responses of neurons in macaque MT. Visual Neuroscience, 13, 87-100.

Britten, K. H., Shadlen, M. N., Newsome, W. T., \& Movshon, J. A. (1993). Responses of neurons in macaque MT to stochastic motion signals. Visual Neuroscience, 10, 1157-1169.

Cauquil, A. S., Edmonds, G. E., \& TAYlor, M. J. (2000). Is the facesensitive N170 the only ERP not affected by selective attention? Cognitive Neuroscience, 11, 2167-2171.

FARAH, M. (1990). Visual agnosia: Disorders of object recognition and what they tell us about normal vision. Cambridge, MA: MIT Press.

Gauthier, I., Tarr, M. J., Anderson, A. W., Skudlarski, P., \& Gore, J. C. (1999). Activation of the middle fusiform face area increases with expertise in recognizing novel objects. Nature Neuroscience, 6, 568-573.

Goffaux, V., Gauthier, I., \& Rossion, B. (2003). Spatial scale contributions to early visual differences between face and object processing. Cognitive Brain Research, 16, 416-424.

Hochstein, S., \& AHISSAR, M. (2002). View from the top: Hierarchies and reverse hierarchies in the visual system. Neuron, 36, 791-804.

JEFFREYs, D. A. (1989). A face-responsive potential recorded from the human scalp. Experimental Brain Research, 78, 193-202.

Kanwisher, N., Stanley, D., \& Harris, A. (1999). The fusiform face area is selective for faces not animals. NeuroReport, 10, 183-187.

Martinez, A., Di Russo, F., Anllo-Vento, L., \& Hillyard, S. A. (2001). Electrophysiological analysis of cortical mechanisms of selective attention to high and low spatial frequencies. Clinical Neurophysiology, 112, 1980-1998.

McCarthy, G., Puce, A., Belger, A., \& Allison, T. (1999). Electrophysiological studies of human face perception: II. Response properties of face-specific potentials generated in occipitotemporal cortex. Cerebral Cortex, 9, 431-444.

O'Craven, K. M., \& Kanwisher, N. (2000). Mental imagery of faces and places activates corresponding stimulus-specific brain regions. Journal of Cognitive Neuroscience, 12, 1013-1023.

OLIVARES, E. I., \& Iglesias, J. (2000). Neural bases of perception and recognition of faces. Revista de Neurologia, 30, 946-952.

Rossion, B., Gauthier, I., Goffaux, V., Tarr, M. J., \& CromMELINCK, M. (2002). Expertise training with novel objects leads to left-lateralized face-like electrophysiological responses. Psychological Science, 13, 250-257.

TANAKA, J. W., \& CURRAN, T. (2001). A neural basis for expert object recognition. Psychological Science, 12, 43-47.

Tong, F., Nakayama, K., Vaughan, J. T., \& Kanwisher, N. (1998). Binocular rivalry and visual awareness in human extrastriate cortex. Neuron, 21, 753-759.

WOJCIUlik, E., KANWISHer, N., \& Driver, J. (1998). Covert visual attention modulates face-specific activity in the human fusiform gyrus: fMRI study. Journal of Neurophysiology, 79, 1574-1578.

\section{NOTES}

1. The ERP averages the EEG response over trials.

2. This was computed by taking the average amplitude in the time window from 140 to $200 \mathrm{msec}$ for each condition.

(Manuscript received January 16, 2003; revision accepted for publication June 20, 2003.) 